\title{
硼基材料在锂硫电池中的研究进展
}

\author{
李高然，李红阳，曾海波 \\ (南京理工大学 材料科学与工程学院, 纳米光电材料研究所, 新型显示材料与器件工信部重点实验室, 南京 210094)
}

摘 要: 锂硫电池因其高能量密度和低成本等优势成为新一代电化学储能技术的重要发展方向。然而, 其较低的转 换反应动力学和可逆性导致电池的实际容量、库仑效率和循环稳定性等仍难以满足实用化发展需求。对此, 合理设 计和开发具有导电、吸附、催化特性的功能材料是稳定和促进硫电化学反应的关键途径。得益于硼独特的原子和 电子结构, 嗍基材料具有丰富且可调的物理、化学和电化学性质, 近年来在锂硫电池的研究中受到了广泛关注。本 文综述了近期嗍基材料, 包括嗍烯、硼原子掺杂碳、金属嗍化物和非金属嗍化物在锂硫电池中的研究进展，总结了 存在的问题并展望了未来的发展方向。

关 键 词: 锂硫电池; 嗍化物; 化学掺杂; 硼烯; 穿梭效应; 综述

中图分类号: TQ174 文献标志码: A

\section{Recent Progress of Boron-based Materials in Lithium-sulfur Battery}

\author{
LI Gaoran, LI Hongyang, ZENG Haibo
}

(MIIT Key Laboratory of Advanced Display Materials and Devices, Institute of Nano Optoelectronic Materials, School of Materials Science and Engineering, Nanjing University of Science and Technology, Nanjing 210094)

\begin{abstract}
Lithium-sulfur (Li-S) batteries play a crucial role in the development of next-generation electrochemical energy storage technology due to its high energy density and low cost. However, their practical application is still hindered by the sluggish kinetics and low reversibility of the conversion reactions, which contribute to relatively low practical capacity, Coulombic inefficiency, and cycling instability. In this regard, the rational design of conductive, adsorptive and catalytic functional materials presents a critical pathway to stabilize and promote sulfur electrochemistry. Benefiting from the unique atomic and electronic structures of boron, boron-based materials exhibit multifarious and tunable physical, chemical and electrochemical properties, and have received extensive research attentions in Li-S batteries. This paper reviews the recent research progress of boron-based materials, including borophene, boron atom-doped carbon, metal borides and non-metal borides in Li-S batteries, concludes the remaining problems and proposes the future developing perspective.
\end{abstract}

Key words: lithium-sulfur battery; boride; chemical doping; borophene; shuttle effect; review

发展绿色可再生能源，开发先进的能源转换和 存储方法，建立高效清洁的能源系统是应对当今世 界能源危机和气候变化的必然选择。以电池为代表
的电化学储能技术，可将新型清洁能源进行转化存 储，并以更高效和便捷的形式加以利用，在推动绿 色能源经济和可持续发展中扮演重要角色 ${ }^{[1-2]}$ 。在诸

收稿日期：2021-03-23；收到修改稿日期：2021-05-07; 网络出版日期：2021-05-25

基金项目：江苏省国际科技合作项目(BZ2020063); 中国博士后面上项目(2021M691586); 江苏省博士后科研资助(2021K446C) Program for International S\&T Cooperation of Jiangsu Province (BZ2020063); China Postdoctoral Science Foundation (2021M691586); Postdoctoral Research Funding Scheme of Jiangsu Province (2021K446C)

作者简介：李高然(1989-), 男, 教授. E-mail: gaoranli@njust.edu.cn LI Gaoran (1989-), male, professor. E-mail: gaoranli@njust.edu.cn 
多电池技术中, 锂离子电池因其较高的能量密度和 无记忆效应等优势, 自 1991 年商业化以来取得了迅 猛发展, 并在电动汽车、便携式电子设备、国防等 领域获得了广泛的应用 ${ }^{[3-4]}$ 。然而, 随着用电设备的 不断发展，传统锂离子电池已难以满足日益增长的 能量需求。在这样的背景下, 锂硫电池因其较高的理 论比容量 $\left(1675 \mathrm{mAh} \cdot \mathrm{g}^{-1}\right)$ 和能量密度 $\left(2600 \mathrm{Wh} \cdot \mathrm{kg}^{-1}\right)$ 引起了广泛关注。同时, 硫资源储量丰富、分布广 泛、价格低廉且环境友好, 使得锂硫电池成为近年 来新型二次电池领域的研究热点 ${ }^{[5-6]}$ 。

\section{1 锂硫电池工作原理以及存在问题}

锂硫电池通常是以单质硫为正极, 金属锂为负 极的电池体系, 基本电池构架如图 1(a)所示。其电 化学反应是一个涉及多电子转移的多步骤转化反应 过程, 并伴随着固-液相转变以及一系列多硫化锂 中间产物(图 1(b) $)^{[7-8]}$ 。其中, 位于反应链两端的单质 硫和短链 $\mathrm{Li}_{2} \mathrm{~S}_{2} / \mathrm{Li}_{2} \mathrm{~S}$ 不溶于电解液, 以沉淀的形式存 在于电极表面; 而长链的多硫化锂 $\left(\mathrm{Li}_{2} \mathrm{~S}_{x}, 4 \leqslant x \leqslant 8\right)$ 在电解液中则具有较高的溶解度和迁移能力。基于 电极材料的本征性质及其固-液相转化反应机制, 锂硫电池在具备能量和成本优势的同时, 也面临许 多问题和挑战 ${ }^{[9-12]}$ :

1)固相的单质硫及 $\mathrm{Li}_{2} \mathrm{~S}$ 在电极表面堆积, 其本
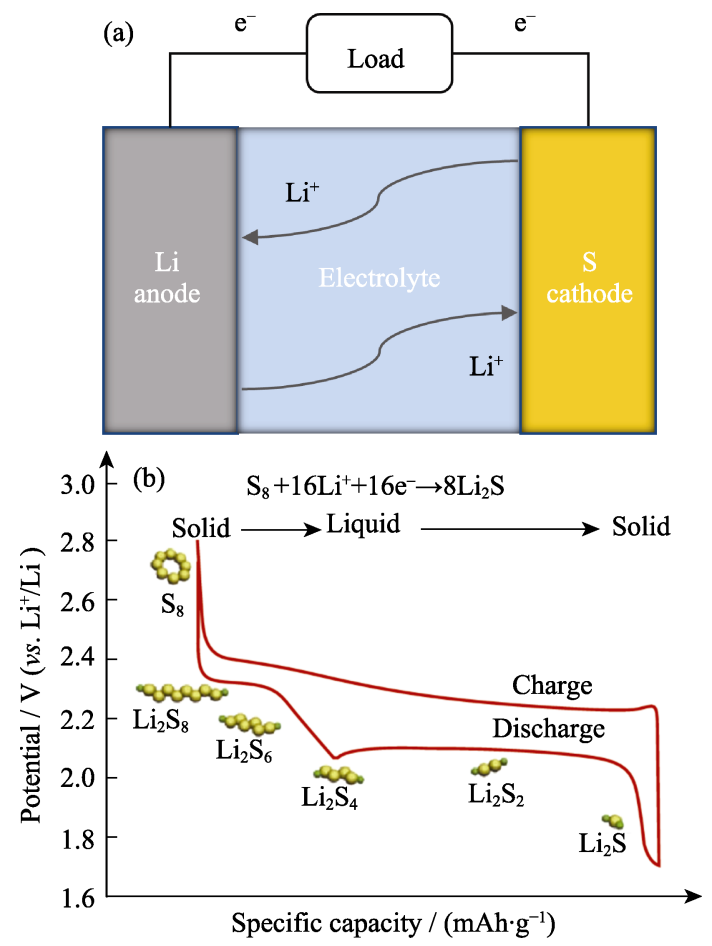

图 1 锂硫电池(a)框架示意图和(b)充放电过程图 ${ }^{[7]}$

Fig. 1 Schematic diagram of (a) lithium-sulfur battery configuration and (b) corresponding charge-discharge process ${ }^{[7]}$
征的电子、离子惰性导致电荷传输困难, 反应动力 学缓慢, 进而降低了活性物质利用率和电池实际 容量。

2)反应链两端的硫和 $\mathrm{Li}_{2} \mathrm{~S}$ 密度差异较大(2.07 vs $1.66 \mathrm{~g} \cdot \mathrm{cm}^{-3}$ ), 材料在反应过程中经历高达 $80 \%$ 的体 积变化, 电极的机械结构稳定性面临巨大的挑战。

3)多硫化锂在电解液中的溶解和迁移行为引发 严重的“穿梭效应”，导致严重的活性物质损失和库 仑损失。此外, 多硫化锂参与负极表面的化学/电化 学副反应，不仅使活性物质进一步损失，同时钝化 和腐蚀了负极表面, 加剧锂枝晶的形成和生长, 增 加了安全隐患。

这些问题相互关联、相互影响，大大增加了电 池系统的复杂性, 导致当前锂硫电池在活性物质利 用率、实际能量密度、循环稳定性和安全性等方面 仍难以满足实际应用需要。通过以上问题分析可以 看出, 硫电化学反应过程的合理管控是提升锂硫电 池性能的必经之路。而如何实现对硫电化学的有效 管理和改善, 则有赖于先进功能材料的针对性设 计、开发和应用。其中, 极具代表性的策略是发展 具有导电、吸附和催化特性的功能材料作为硫正极 宿主或修饰隔膜, 通过其与多硫化锂的物理化学相 互作用将活性物质限域在正极区域，抑制溶解和扩 散, 并促进其电化学转换, 从而缓解穿梭效应, 提 升电池的能量效率和循环稳定性 ${ }^{[13-14]}$ 。围绕该思路, 研究人员针对性地开发了各类功能材料, 包括碳材 料、导电聚合物、金属有机框架、金属氧化物/硫化 物/氮化物等，取得了不错的成效 ${ }^{[15-19]}$ 。

\section{2 硼基材料在锂硫电池中的应用}

嗍是最小的类金属元素, 较小的原子半径和较 大的电负性使其容易形成带有金属性的共价化合 物。嗍原子具有典型的缺电子结构, 其价电子排布 为 $2 \mathrm{~s}^{2} 2 \mathrm{p}^{1}$, 可通过多种杂化形式与其他原子共用一 个或多个电子形成多中心键 ${ }^{[20-21]}$ 。这些特性使得嗍 化物结构具有很强的可调性, 表现出独特而丰富的 化学和物理性质, 可广泛应用于轻工、建材、国防、 能源等诸多领域 ${ }^{[22-23]}$ 。相比而言, 嶰基材料在锂硫 电池中的研究仍处于起步阶段。近年来纳米技术和 表征方法不断进步，不断挖掘和开发硼基材料的结 构特性, 使其在锂硫体系中的针对性研究和应用也 开始崭露头角。鉴于此, 本文围绕硼烯、嗍原子掺 杂碳、金属硼化物和非金属硼化物等典型的硣基材 料, 综述了其在锂硫电池中的最新研究进展, 总结 了现存问题并展望了今后的发展方向。 


\section{1 硼烯}

作为硼单质中极具代表性的一类同素异形体， 嗍烯具有类石墨烯的单原子厚度二维结构, 与体相 硼单质相比表现出更加优越的电学、力学、热学性 质, 是二维材料中的后起之秀 ${ }^{[24]}$ 。基于硼原子排布 的拓扑差异, 硼烯具有丰富的晶体结构和电子特性, 以及各向异性的导电性质。从图 2(a,b) 可以看出, 嗍 烯中电子倾向集中于嗍原子顶部, 这些电子极化区 域具有较高的成键活性, 有望在锂硫电池体系中为 多硫化物提供良好的化学吸附位点 ${ }^{[25]}$ 。同时, 嗍烯 薄膜具有良好的导电性和物理化学稳定性, 因此在 锂硫电池中具有较好的应用潜力。

Jiang 等 ${ }^{[26]}$ 通过理论计算发现, 嗍烯对多硫化 锂表现出很强的吸附能力, 然而该强相互作用也容 易引发 Li-S 团簇分解, 造成活性物质硫的损失。与 之相比, 具备本征缺陷结构的嗍烯表面对多硫化锂 的吸附更加温和 ${ }^{[27]}$, 使其在限制穿梭行为的同时可 避免环状结构的分解和破坏, 有望成为更加合适的 多硫化锂吸附材料。同时, 嗍烯-多硫化锂吸附结构 的能带分析结果表明该吸附团簇呈现金属性, 这主 要得益于硼稀本征的金属性特征及其较强的电声耦 合强度, 有望帮助硫的电化学转换过程获得更好的 反应动力学 ${ }^{[28]}$ 。此外, Grixti 等 ${ }^{[29]}$ 模拟了多硫化锂分 子在 $\beta_{12}$-硼烯表面的扩散过程, 发现 $\beta_{12}$-硼烯对一系 列多硫化锂均表现出较强的吸附; 而 $\mathrm{Li}_{2} \mathrm{~S}_{6}$ 和 $\mathrm{Li}_{2} \mathrm{~S}_{4}$ 分子在 armchair 方向的最低扩散能垒分别为 0.99 和
$0.61 \mathrm{eV}$, 相比在 zigzag 方向上的扩散更加容易。得 益于良好的吸附能力和适中的扩散能垒, $\beta_{12}$-硼烯被 认为是优秀的多硫化锂吸附材料, 有望抑制锂硫电 池中的穿梭效应，提升硫电化学反应可逆性。

然而, 目前硼稀在锂硫电池中的研究大多仍停 留在理论预测阶段, 实验上的印证鲜有报道。这主 要归各于嗍稀的制备仍存在较大难度。在二十世纪 九十年代就已预测嗍稀的存在, 但直到 2015 年才真 正制备获得 ${ }^{[30]}$ 。部分原因可能是由于硼只有三个价 电子, 需通过形成框架结构来补偿缺失电子, 更容 易形成 3D 而非 2D 结构。目前嗍稀的制备通常需依 赖分子束外延等技术以及高真空、高温等条件, 合 成门槛较高 ${ }^{[31]}$ 。因此, 需要开发更加简单、高效的 嗍稀合成方法, 并进一步实验探索和论证其在锂硫 电池中的效果和相关机制。

\section{2 硼原子掺杂碳}

化学掺杂碳材料是新能源研究领域的热点材 料。适当的元素掺杂可保留碳材料轻质和高电导等 优势, 同时赋予其额外的物理和化学特性, 从而适 配不同的应用场景 ${ }^{[32-33]}$ 。化学掺杂碳材料在锂硫电 池中的研究十分广泛 ${ }^{[34-35]}$, 其中以氮原子等强电负 性原子掺杂较为常见。相比之下, 嗍属于缺电子结 构, 电负性比碳更低, 掺入碳晶格之后显正电性, 有望对带负电的多硫阴离子形成良好的吸附效果, 从而缓解穿梭效应 ${ }^{[36-37]}$ 。

Yang 等 ${ }^{[38]}$ 以硼掺杂多孔碳作为硫正极宿主材 (a)
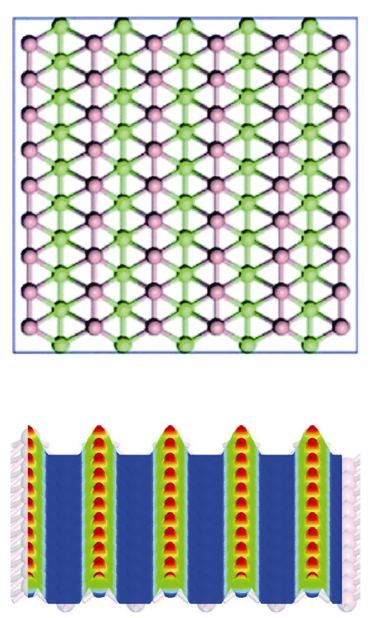

Triangular borophene $(\Delta \mathrm{B})$
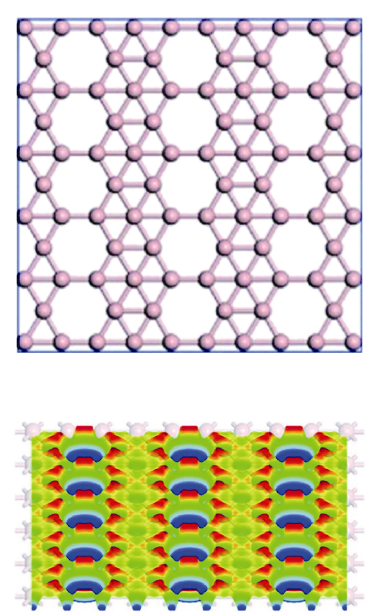

$\beta_{12}$-borophene $\left(\beta_{12} \mathrm{~B}\right)$

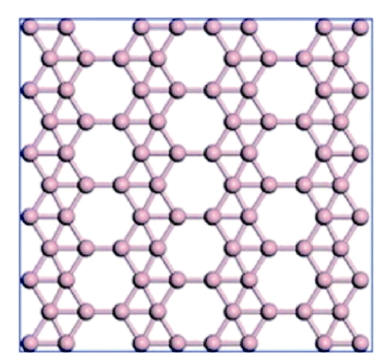

(b)
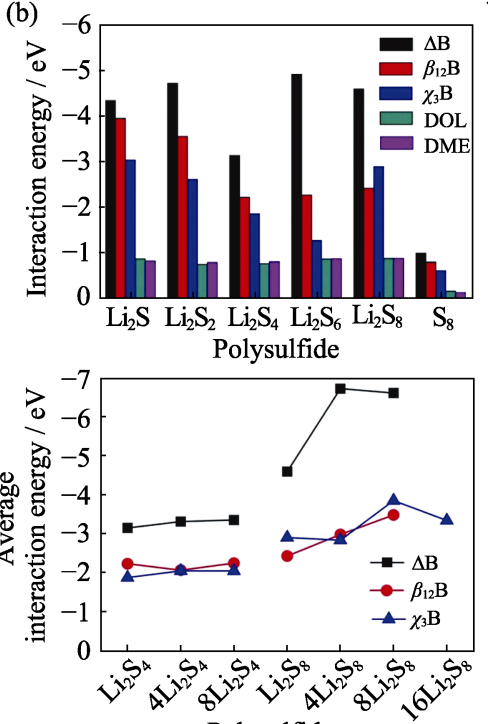

Polysulfide

图 2 (a)不同喼稀结构模型及对应电荷密度分布; (b)多硫化物在不同嗍稀上的吸附能 ${ }^{[25]}$

Fig. 2 (a) Structural models of different borophenes and their corresponding charge density distributions,

(b) adsorption energies of polysulfides on different borophenes ${ }^{[25]}$

DOL: 1,3-dioxolane; DME: Dimethoxyethane; Colorful figures are available on website 
料, 发现硼掺杂不仅提高了碳材料的电子电导率, 而且诱导碳基质发生正极化, 通过静电吸附以及路 易斯相互作用有效吸附和针定了带负电的多硫离子, 从而抑制其溶解扩散(图 3(a,b))。因此, 基于硼掺杂 多孔碳的硫正极相比纯碳以及氮掺杂的样品表现出 更高的初始容量和更稳定的循环性能。Xu 等 ${ }^{[39]}$ 通 过水热一锅法获得了硼原子掺杂的碳纳米管/硫的 复合正极材料(BUCNTs/S)。液相原位合成使硫在复 合物中分布更加均匀, 而硼掺杂使碳基宿主材料具 有更高的电导率和更强的固硫能力。所得 BUCNTs/S 电极在 $0.2 C$ 下获得了 $1251 \mathrm{mAh} \cdot \mathrm{g}^{-1}$ 的初始容量, 并 在循环 400 圈后仍可保持在 $750 \mathrm{mAh} \cdot \mathrm{g}^{-1}$ 。除了硫正 极宿主, 嗍掺杂碳材料在电池功能隔膜的设计中也 扮演了重要的角色。Han 等 ${ }^{[40]}$ 将轻质的硼掺杂石墨 烯涂覆在传统隔膜上构建了功能修饰层, 利用其对 多硫化物的吸附和再利用, 有效缓解了穿梭效应并 提高了活性物质利用率。

鉴于不同掺杂元素的基本性质，及其在碳晶格结 构中不同的作用方式, 多元素共掺杂是调控碳材料 表面化学, 改善硫电化学反应的重要策略之一 ${ }^{[41-43]}$ 。 对此, Kuang 课题组 ${ }^{[44]}$ 通过水热法首次合成了氮、硼 共掺杂的石墨烯纳米带(NBCGNs)作为硫正极的宿
主材料, 如图 3(c)所示。研究发现, 氮、嗍共掺杂的 协同效应不仅诱导 NBCGNs 获得更大的比表面积、 孔体积和更高的电导率, 而且还有助于硫在正极中 均匀分布。更重要的是, 共掺杂体系中嗍和氮作为 缺电子和富电子中心, 可分别与 $\mathrm{S}_{x}{ }^{2-}$ 和 $\mathrm{Li}^{+}$通过路易 斯相互作用进行键合, 从而更高效地吸附多硫化锂, 显著提升电池的循环和倍率性能(图 3(d, e))。基于类 似的高、低电负性元素搭配掺杂策略, Jin 等 ${ }^{[45]}$ 以嗍 酸为掺杂剂制备了嗍、氧共掺杂的多壁碳纳米管宿 主材料, 所得电池循环 100 圈后比容量仍保持 $937 \mathrm{mAh} \cdot \mathrm{g}^{-1}$, 显著优于基于普通碳管的电池性能 $\left(428 \mathrm{mAh} \cdot \mathrm{g}^{-1}\right)$ 。此外, 研究人员亦尝试了其他共掺 杂形式，包括嗍硅共掺杂石墨烯 ${ }^{[46]}$ 、钴金属和硼氮 共掺杂石墨烯 ${ }^{[47]}$ 等, 均有效提升了电池性能, 而其 中共掺杂组分的协同效应对改善硫电化学反应起到 至关重要的作用。

硼元素掺杂可有效提升碳材料的本征导电性以 及表面化学极性, 强化化学吸附抑制多硫化锂的穿 梭行为, 从而改善硫电化学反应动力学和稳定性, 提升电池性能。尽管如此, 嗍掺杂碳材料在锂硫电 池的研究中仍存在许多问题, 有待进一步的挖掘和 解析。比如，硼掺量以及掺杂构型对碳材料导电性、

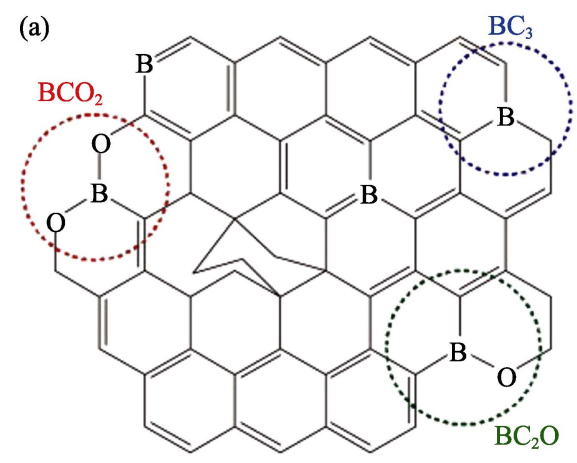

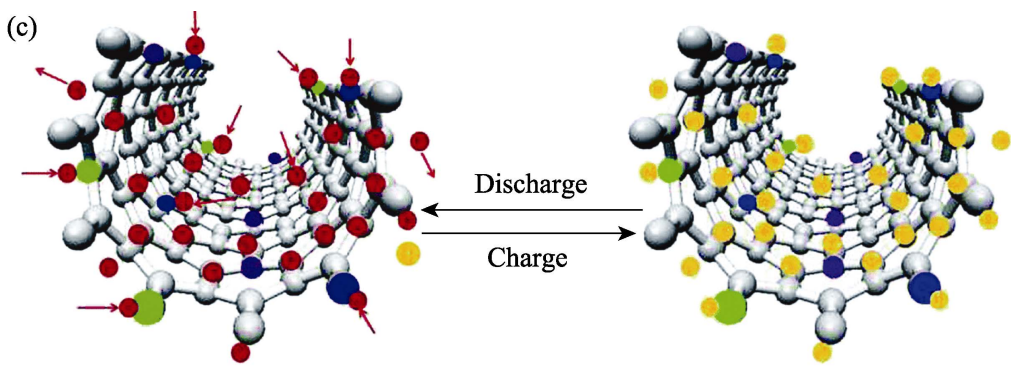

Oxygen-containing groups Boron atoms Sulfur
Nitrogen atoms $\quad$ NBCGN/S composite Polysulfides
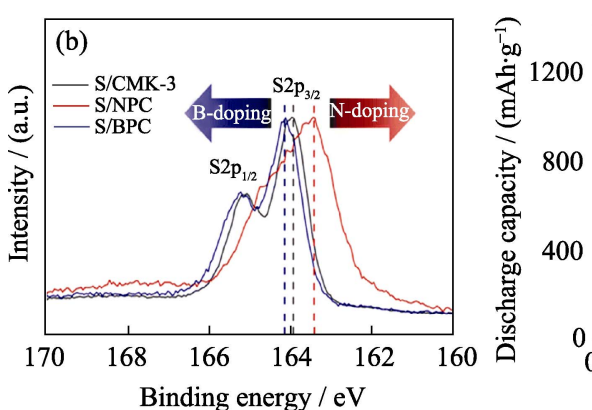

(d)

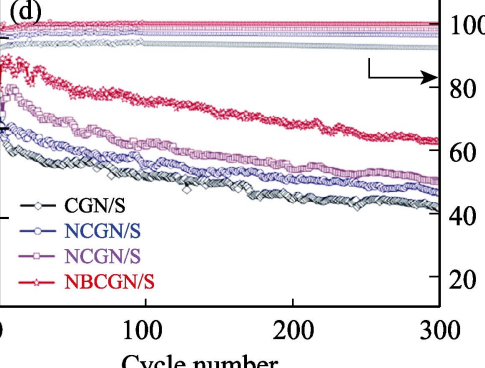

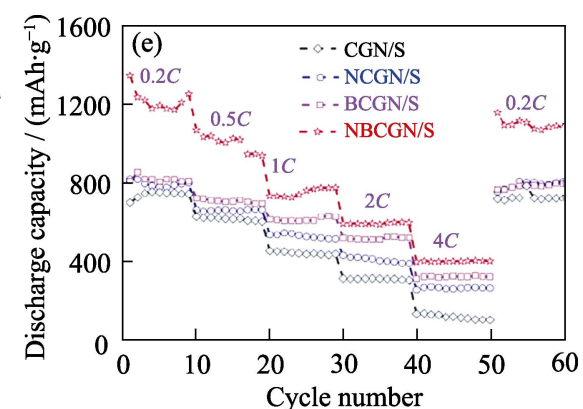

图 3 (a)硼掺碳骨架结构示意图, (b)基于不同掺杂的硫碳复合物的 S2p XPS 谱图 ${ }^{[38]}$; (c)NBCGN/S 充放电过程示意图, 不同元素掺杂碳管/硫的复合电极(d)在 $0.2 C$ 下的循环性能以及(e)倍率性能 ${ }^{[44]}$

Fig. 3 (a) Scheme of B-doped carbon backbone, (b) S2p XPS spectra of sulfur composites based on different element-doped porous carbon; and (c) scheme of charge-discharge process of NBCGN/S composite, (d) cycling at $0.2 C$ and (e) rate performances of sulfur electrodes based on different element-doped curved graphene nanoribbons ${ }^{\text {[44] }}$ NPC: N-doped porous carbon, BPC: B-doped porous carbon; CGN: curved graphene nanoribbons; NCGN: N-doped CGN; BCGN: B-doped CGN; NBCGN: N, B-codoped CGN; Colorful figures are available on website 
表面电荷分布以及对多硫化锂吸附行为的影响。同 时, 如何获得高硼掺杂量的碳材料以及如何精确调 控掺杂构型, 均有赖于开发先进的制备方法和技 术。此外, 对于多元素共掺杂体系, 仍需要进一步探 索更合适的掺杂元素搭配, 建立系统性的构效关系, 厘清共掺杂结构的协同效应机制及其对硫电化学中 主客体相互作用方式和强度的影响。

\section{3 金属嗍化物}

金属化合物由于其本征的化学极性特征以及良 好的形貌结构可塑性, 一直都是锂硫电池功能材料 的研究热点。与常见的金属氧化物、硫化物、氮化 物等离子型化合物不同, 金属嗍化物通常由嗍和金 属元素基于共价键构成, 其填充型结构继承了部分 金属性, 表现出远高于其他金属化合物的导电性

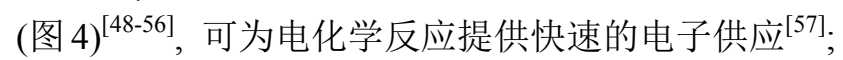
同时, 金属与硼之间存在局域有限的离子键极性结 构, 可为多硫化物提供良好的吸附位点 ${ }^{[58-59]}$; 此外, 高负电性的嗍和过渡金属合金化后稳定性减弱, 更 容易参与氧化还原反应, 这使得金属㸴化物有可能 通过表面反应作为媒介, 参与锂硫电化学反应 ${ }^{[60]}$ 。

Guan 等 ${ }^{[61]}$ 利用液相还原法在石墨烯上负载无 定形的 $\mathrm{Co}_{2} \mathrm{~B}$ 纳米颗粒制备了硫正极的宿主材料。 研究发现, 嗍和钴均可作为吸附位点, 化学针定多 硫化锂, 从而抑制其溶解迁移。配合石墨烯优异的 长程导电性, 电池在 $1 C$ 倍率下循环 450 圈后放电比 容量仍有 $758 \mathrm{mAh} \cdot \mathrm{g}^{-1}$, 每圈的容量衰减率为 $0.029 \%$, 表现出优异的循环性能。基于类似的协同吸附效应, $\mathrm{Co}_{2} \mathrm{~B} @ \mathrm{CNT}$ 复合材料作为锂硫电池功能隔膜对 $\mathrm{Li}_{2} \mathrm{~S}_{6}$ 的吸附能力高达 $11.67 \mathrm{mg} \cdot \mathrm{m}^{-2[62]}$, 可有效阻挡 多硫化物扩散渗透, 达到抑制穿梭效应的目的。在 此基础上, Guan 等 ${ }^{[63]}$ 进一步以二维金属碳化物 (MXene)作为载体, 制备了 $\mathrm{Co}_{2} \mathrm{~B} @ \mathrm{MX}$ ene 的异质结 复合材料(图 5(a d ) )。通过理论计算发现, 异质结界 面处的电子相互作用导致电子从 $\mathrm{Co}_{2} \mathrm{~B}$ 向 MXene 转

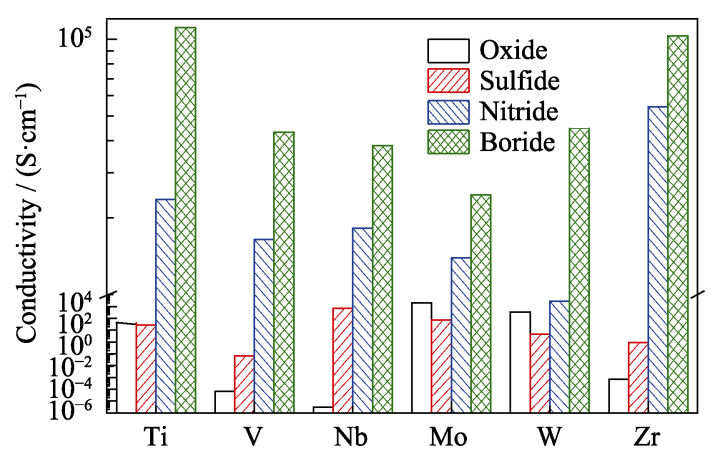

图 4 几类金属化合物导电性的对比 ${ }^{[48-56]}$

Fig. 4 Conductivity comparison with several categories of metal compounds ${ }^{[48-56]}$
移，该效应提升了 $\mathrm{Co}_{2} \mathrm{~B}$ 对多硫化物的吸附和催化 能力(图 5(a, b))。因此, 基于 $\mathrm{Co}_{2} \mathrm{~B} @ \mathrm{MX}$ Ne 功能修 饰隔膜的电池在 2000 圈循环过程中的容量衰减率 仅为每圈 $0.0088 \%$, 并且在 $5.1 \mathrm{mg} \cdot \mathrm{cm}^{-2}$ 的硫载量下, 面比容量依然高达 $5.2 \mathrm{mAh} \cdot \mathrm{cm}^{-2}$ (图 5(c, d))。需要 注意的是, 相比晶相结构, 这类非晶相金属嗍化物 材料虽然在材料制备上更加温和简单，但是其原子 分子结构可控性和稳定性相对欠佳, 这对明晰其组 分和微观结构, 以及探究其对硫电化学反应过程的 影响机制构成了不小的阻碍。

$\mathrm{TiB}_{2}$ 是经典的金属嗍化物，具有优异的导电性 $\left(\sim 10^{6} \mathrm{~S} \cdot \mathrm{cm}^{-1}\right)$, 在导电陶瓷、精密加工以及电化学器 件等领域应用广泛。 $\mathrm{TiB}_{2}$ 属于典型的六方结构, 具 有较高的硬度和结构弹性, 有助于适应硫反应的体 积变化, 同时其表面大量的不饱和结构有望与多硫 化锂形成较强的界面化学相互作用 ${ }^{[64]}$, 从而达到良 好的吸附和限域效果。 $\mathrm{Li}$ 等 ${ }^{[65]}$ 率先报道了 $\mathrm{TiB}_{2}$ 用作 硫正极的宿主材料, 如图 $5(\mathrm{e} \sim \mathrm{g})$ 所示, 在与 $\mathrm{S}$ 的热 复合过程中, $\mathrm{TiB}_{2}$ 表面被部分硫化, 并通过范德华 力以及路易斯酸碱相互作用有效吸附反应过程中产 生的多硫化锂, 而该作用机制在(001)面上的效果更 加显著。所得硫正极获得 $1 C$ 倍率下 500 圈的稳定 循环, 同时在 $3.9 \mathrm{mg} \cdot \mathrm{cm}^{-2}$ 的硫载量下循环 100 圈后 面比容量仍保留 $3.3 \mathrm{mAh} \cdot \mathrm{cm}^{-2}$, 表现出良好的电化 学性能(图 5(h,i))。基于 XPS 分析和理论计算等结 果, $\mathrm{TiB}_{2}$ 优秀的多硫化锂吸附效果应归功于其表面 “钝化”机制。此外, $\mathrm{Lu}$ 课题组 ${ }^{[66]}$ 对比了 $\mathrm{TiB}_{2} 、 \mathrm{TiC}$ 和 $\mathrm{TiO}_{2}$ 对多硫化锂的吸附效果, 并探究了相应化学 吸附和溶剂化脱附之间的竞争机制。结果表明, 电 负性较低的嗍使得 $\mathrm{TiB}_{2}$ 具有更强的吸附能力, 配合 溶剂化能力较弱的醚类电解液, 可有效提高硫利用 率, 增强电化学反应可逆性。鉴于此, $\mathrm{TiB}_{2}$ 亦被用于 构建多功能隔膜 ${ }^{[67]}$, 高效吸附针定和再利用活性物 质, 显著提升了电池循环稳定性, $0.5 \mathrm{C}$ 循环 300 圈 后容量可以保持初始值的 $85 \%$ 。

与 $\mathrm{TiB}_{2}$ 相似, $\mathrm{MoB}$ 具有良好的导电性, 同时其 本征的二维结构有利于充分暴露吸附位点, 有望成 为良好的硫正极催化剂 ${ }^{[68]}$ 。克萨斯大学奥斯汀分校 的 Manthiram 课题组 ${ }^{[69]}$ 以 $\mathrm{Sn}$ 为还原剂, 通过固相法 合成了 $\mathrm{MoB}$ 纳米颗粒, 对多硫化锂表现出良好的 吸附和催化能力。MoB 具有较高的电子电导率 $\left(1.7 \times 10^{5} \mathrm{~S} \cdot \mathrm{m}^{-1}\right)$, 可为硫的反应提供快速的电子供 应; 同时, $\mathrm{MoB}$ 的亲水表面性质有利于电解液润湿, 帮助锂离子快速传输, 从而保障了贫电解液条件下 的活性物质利用率; 此外, 纳米化的 $\mathrm{MoB}$ 可以充分 暴露由缺电子嗍原子所诱导的催化活性位点, 使材料 

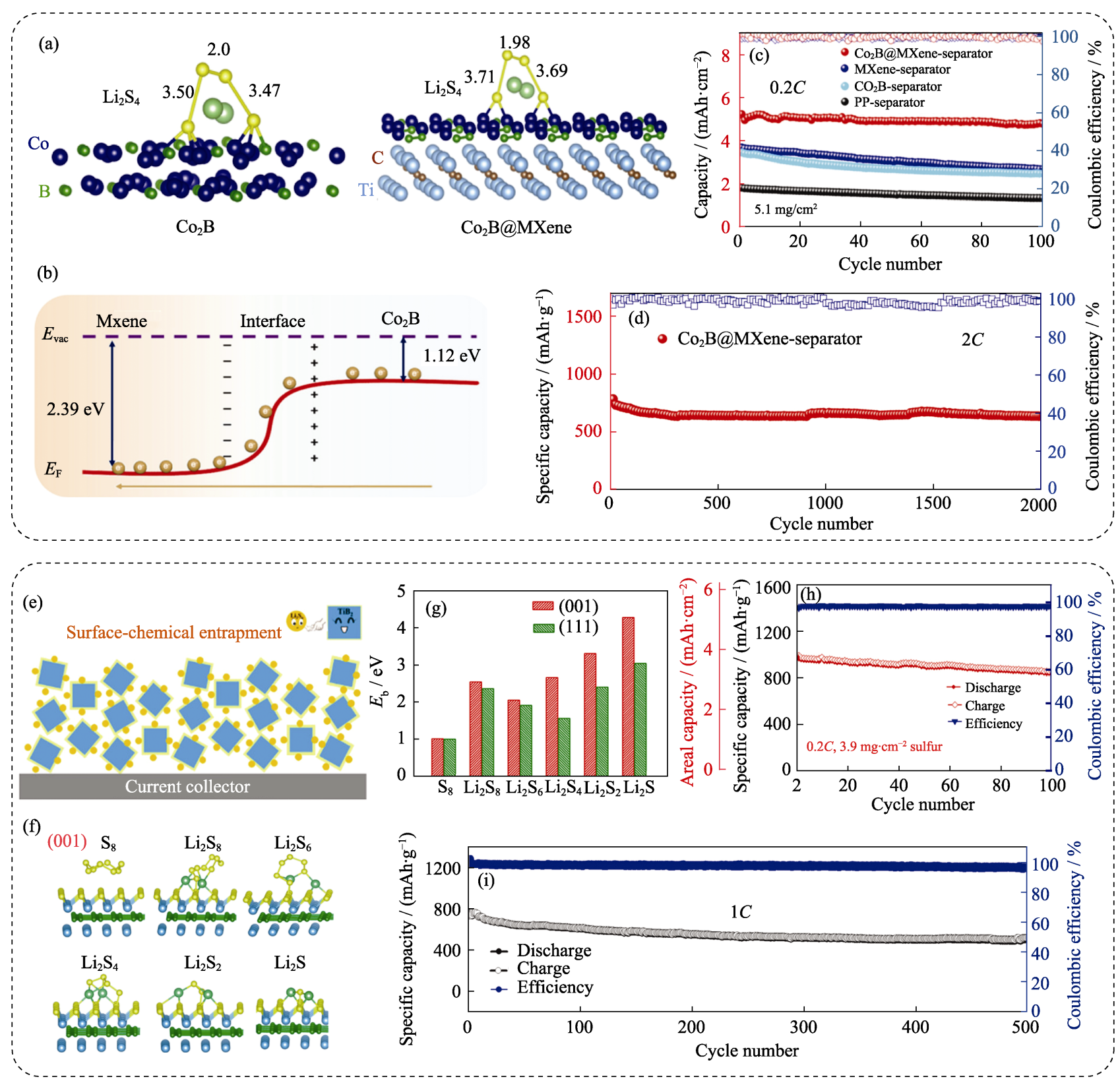

图 5 (a) $\mathrm{Li}_{2} \mathrm{~S}_{4}$ 在 $\mathrm{Co}_{2} \mathrm{~B}$ 和 $\mathrm{Co}_{2} \mathrm{~B} @ \mathrm{MXene}$ 上的吸附构型, (b)Co $\mathrm{Co}_{2} \mathrm{~B}$ 和 $\mathrm{MXene}$ 界面处的电子重排示意图, $(\mathrm{c}) \mathrm{Co}_{2} \mathrm{~B} @ \mathrm{MXene}$ 和其他 隔膜电池的循环性能对比, (d) $\mathrm{Co}_{2} \mathrm{~B} @ \mathrm{MXene}$ 电池的长循环性能 ${ }^{[63]}$; (e) $\mathrm{TiB}_{2}$ 表面固硫作用示意图, (f)不同硫物种在硫化 $\mathrm{TiB}_{2}$ 的 $(001)$ 面上的吸附构型及 $(\mathrm{g})$ 在 $(001)$ 和(111)面上的吸附能 $\left(E_{\mathrm{b}}\right)$ 对比，基于 $\mathrm{TiB}_{2}$ 硫正极的(h)高载量和(i)长循环性能 ${ }^{[63,65]}$

Fig. 5 (a) $\mathrm{Li}_{2} \mathrm{~S}_{4}$ adsorption configurations on $\mathrm{Co}_{2} \mathrm{~B}$ and $\mathrm{Co}_{2} \mathrm{~B} @ \mathrm{MXene}$ surfaces, (b) scheme of the electron redistribution at the interfaces between $\mathrm{Co}_{2} \mathrm{~B}$ and MXene, (c) cycling performances of cells based on $\mathrm{Co}_{2} \mathrm{~B} @ \mathrm{MXene}$ and other separators,

(d) long-term cycling performance of the $\mathrm{Co}_{2} \mathrm{~B} @ \mathrm{MXene}$ cell ${ }^{[63]}$; (e) schematic illustration of surface-chemical entrapment of polysulfides on $\mathrm{TiB}_{2}$, (f) adsorption configurations and (g) energies of sulfur species on (001) and (111) surfaces of $\mathrm{TiB}_{2}$, (h) high-loading performance and (i) long-term cycling of $\mathrm{TiB}_{2}$-based sulfur electrode ${ }^{[63,65]}$ PP: polypropylene; Colorful figures are available on website

同时拥有优异的本征和表观催化活性。基于这些优 势, MoB 即使添加量较小, 也能显著提升电化学性 能, 表现出可观的实用性。所得电池在 $1 C$ 倍率下循 环 1000 圈容量衰减每圈仅为 $0.03 \%$; 且在硫载量 $3.5 \mathrm{mg} \cdot \mathrm{cm}^{-2}$ 和电解液/硫比例 $(\mathrm{E} / \mathrm{S}) 4.5 \mathrm{~mL} \cdot \mathrm{g}^{-1}$ 下, 实 现了优秀的软包装电池循环性能。此外, Nazar 课题 组 ${ }^{[70]}$ 采用轻质的 $\mathrm{MgB}_{2}$ 作为多硫化锂电化学转化介
质，发现 $\mathrm{B}$ 和 $\mathrm{Mg}$ 均可作为多硫阴离子的吸附位点, 强化电子转移, 获得了高硫载量 $\left(9.3 \mathrm{mg} \cdot \mathrm{cm}^{-2}\right)$ 下较 好的循环稳定性。

这些工作充分说明了金属嗍化物对改善硫电化 学反应的有效性和优越性。然而，与金属氧化物、 硫化物等体系相比，金属嗍化物在锂硫电池中的研 究报道仍相对较少, 对材料和相关机制研究亦有待 
扩充和深入。此外, 晶相金属嗍化物通常结构强度 较高, 制备过程需跨越较高的能垒, 涉及高温、高压 等苛刻条件, 限制了其研究和应用。因此, 开发简 单、温和、高效的金属嗍化物合成方法亦是金属硓 化物研究的重要方向。

\section{4 非金属磁化物}

与金属砋化物相比, 非金属硼化物通常密度更 小、质量更轻, 有利于开发高能量密度电池; 然而其 较低的导电性则对硫电化学反应效率和动力学造成 阻力。目前, 研究人员围绕非金属硼化物包括氮化

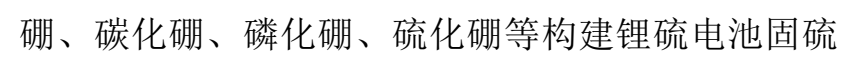
材料取得了一定进展 ${ }^{[71-73]}$ 。

氮化硼 $(\mathrm{BN})$ 和碳化硼 $(\mathrm{BC})$ 是两类最具代表性, 也是研究较为广泛的非金属嗍化物。 $\mathrm{BN}$ 是由氮原 子和嗍原子交替连接而成, 主要包括六方、三方、 立方和纤铅矿四种晶型 ${ }^{774]}$ 。其中, 六方氮化硼(h-BN) 由于其类石墨二维结构和局域的电子极化特性, 表 现出宽带隙、高导热以及良好的热稳定性和化学稳 定等特点 ${ }^{[75-76]}$ 。 B-N 结构的极性特征明显, 对多硫 化锂具有较强的化学吸附能力; 同时该表面化学特 征可通过元素掺杂以及拓扑缺陷构建进行调控, 在 保证多硫分子结构稳定的同时提高对其的吸附强 度 ${ }^{[77]}$ 。基于该思路, $\mathrm{Yi}$ 等 ${ }^{[78]}$ 报道了一种贫氮的少层 氮化嗍 (v-BN)作为硫正极的宿主材料(图 6(a)), 研 究发现, v-BN 中的正电性空位不仅有助于固定和转 化多硫化物, 而且还加速了锂离子的扩散迁移。与 原始 $\mathrm{BN}$ 相比, 基于 $\mathrm{v}$-BN 的正极在 $0.1 C$ 下具有更 高的初始容量(1262vs $\left.775 \mathrm{mAh} \cdot \mathrm{g}^{-1}\right)$, 且在 $1 C$ 下循 环 500 圈容量衰减率仅为每圈 $0.084 \%$, 表现出良好 的循环稳定性。此外, $\mathrm{He}$ 等 ${ }^{[79]}$ 发现 $\mathrm{O}$ 掺杂可进一步 提升 BN 表面化学极性, 诱导材料形成更大的比表 面积, 并同步提升本征及表观吸附性能。

虽然 $\mathrm{BN}$ 材料具有较好的化学吸附特性, 但其 本身的导电性较差, 不利于反应电荷转移。因此, 与 导电材料的复合结构设计是进一步提升其综合吸附 和催化性能的重要途径。鉴于此, Deng 等 ${ }^{[80]}$ 设计了 基于类石墨氮化碳 $\left(\mathrm{g}-\mathrm{C}_{3} \mathrm{~N}_{4}\right) 、 \mathrm{BN}$ 和石墨烯的复合型 离子篮作为锂硫电池多功能中间层(图 6(b))。其中, $\mathrm{g}-\mathrm{C}_{3} \mathrm{~N}_{4}$ 结构中 $0.3 \mathrm{~nm}$ 大小的有序离子通道可有效 阻挡多硫化物并允许锂离子通过, BN 作为反应催化 剂促进多硫化物转化, 石墨烯则作为内置集流体提 供优异的长程导电性。得益于这三种二维组分的协 同效应, 所得电池在 $6 \mathrm{mg} \cdot \mathrm{cm}^{-2}$ 的高硫载量以及 $1 C$ 倍率下可稳定循环 500 圈以上(图 6(c))。此外, 研究 人员尝试以更加简单直接的形式, 在正极表面涂覆
一层轻薄的 $\mathrm{BN}$ 纳米片/石墨烯复合薄膜作为保护 层 ${ }^{[11-82]}$, 有效抑制了多硫化锂的溶出扩散, 显著提 升硫正极的比容量和循环稳定性, 在 $3 C$ 下循环 1000 圈过程中容量衰减率仅为每圈 $0.0037 \%$ 。有趣 的是, 汉阳大学的 Ungyu Paik 课题组 ${ }^{[83]}$ 采用另一种 组合思路, 构建了 BN/Celgard/碳三明治结构的多功 能隔膜。如图 6(d) 所示, 碳质层和 BN 层分别涂覆 于普通隔膜的正极侧和负极侧。其中, 碳层和 $\mathrm{BN}$ 层可共同阻挡多硫化锂的穿梭, 限制其扩散到负极 表面, 同时负极侧的 $\mathrm{BN}$ 层亦对锂枝晶的生长起到 限制作用。得益于该协同保护机制, 电池在 $0.5 C$ 下 250 圈循环后具有较高的容量保持率 $(76.6 \%)$ 和比容 量 $\left(780.7 \mathrm{mAh} \cdot \mathrm{g}^{-1}\right)$, 显著优于普通隔膜和纯碳质改 性隔膜(图 6(e))。

与 $\mathrm{N}$ 相比, $\mathrm{C}$ 的电负性更低, 因此 $\mathrm{B}$ 与 $\mathrm{C}$ 电负 性差异较小, 导致 B-C 结构相比 $\mathrm{N}-\mathrm{C}$ 的化学极性更 弱, 但同时 B-C 结构中的电子离域性得到增强, 导 电性更佳 ${ }^{[84-85]}$ 。因此, BC 总体上表现出与 $\mathrm{BN}$ 相对 互补的理化性质, 其密度低、导电性相对较好, 且具 有不错的催化性能，在能源领域颇具应用前景 ${ }^{[86]}$ 。 Luo 等 ${ }^{[87]}$ 在碳纤维上原位生长碳化硼纳米线 $\left(\mathrm{B}_{4} \mathrm{C} @ \mathrm{CNF}\right.$ ) 作为正极宿主材料(图 6(f $\left.\sim \mathrm{h}\right)$ )。其中, $\mathrm{B}_{4} \mathrm{C}$ 通过 $\mathrm{B}-\mathrm{S}$ 成键高效地吸附和限域多硫化物, 同 时其碳纤维导电网络帮助所吸附的硫快速转化, 提 升反应动力学。所得硫正极在 500 次循环后具有 $80 \%$ 的容量保持, 并在高硫含量(质量分数 $70 \%$ ) 和 载量 $\left(10.3 \mathrm{mg} \cdot \mathrm{cm}^{-2}\right)$ 下亦可实现稳定循环。Song 等 ${ }^{[88]}$ 围绕 $\mathrm{B}_{4} \mathrm{C}$ 构建了超强限域作用的硫宿主结构。该结 构以活化的多孔棉织物碳为柔性基质, $\mathrm{B}_{4} \mathrm{C}$ 纳米纤 维则作为活性骨架, 同时还原氧化石墨烯作进一步 包覆, 高效结合了物理和化学的限域作用, 缓解了 活性物质损失, 获得了优异的循环稳定性。鉴于 $\mathrm{B}_{4} \mathrm{C}$ 良好的吸附和催化特性, Zhao 课题组 ${ }^{[89]}$ 通过原位催 化辅助生长的方法将 $\mathrm{B}_{4} \mathrm{C}$ 纳米颗粒均匀分布在碳纤 维布中, 以此高效地分散和暴露活性位点, 所得硫 正极在 $3.0 \mathrm{mg} \cdot \mathrm{cm}^{-2}$ 载量下, 具有高达 $1415 \mathrm{mAh} \cdot \mathrm{g}^{-1}$ $(0.1 C)$ 的初始容量以及 $1 C$ 下 3000 圈的超长寿命, 表 现出良好的应用前景。

由上可知，非金属嗍化物对多硫化锂具有较好 的吸附催化效果, 但是其电导率相对较低, 仍需导 电载体辅助硫电化学反应。其中, 相邻的 $\mathrm{N}$ 和 $\mathrm{C}$ 的 原子电子结构差异使 $\mathrm{BN}$ 和 $\mathrm{BC}$ 材料在导电性以及 与多硫化锂的相互作用方面各具优劣。鉴于此, 结

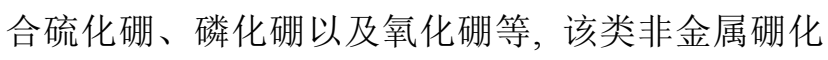
物可作为研究局域化学极性结构与吸附催化能力间 
构效关系的良好载体和平台, 有望通过进一步系统 性的关联和解析, 帮助理解相关微观反应过程、调 控材料精细结构以及提升电池电化学性能。此外, 非金属硼化物在锂硫电池中的进一步应用发展，仍
需依赖其制备方面的改良和优化，发展简单温和的 制备技术，同时开发更高本征电导的材料结构以及 设计更高效的复合材料，平衡和兼顾导电、吸附和 催化效果。
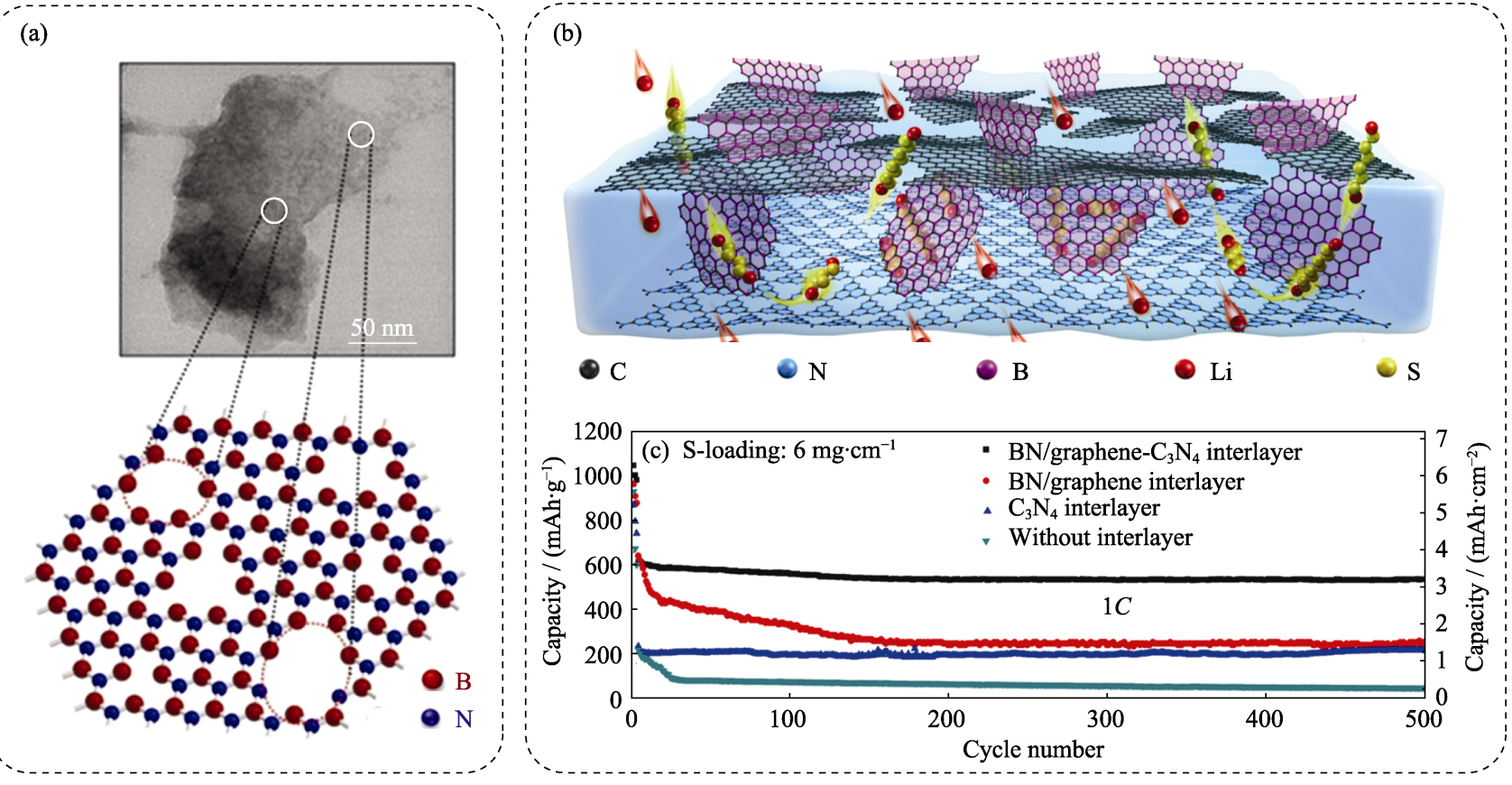

(d) Sulfur cathode BN-carbon separator Li metal
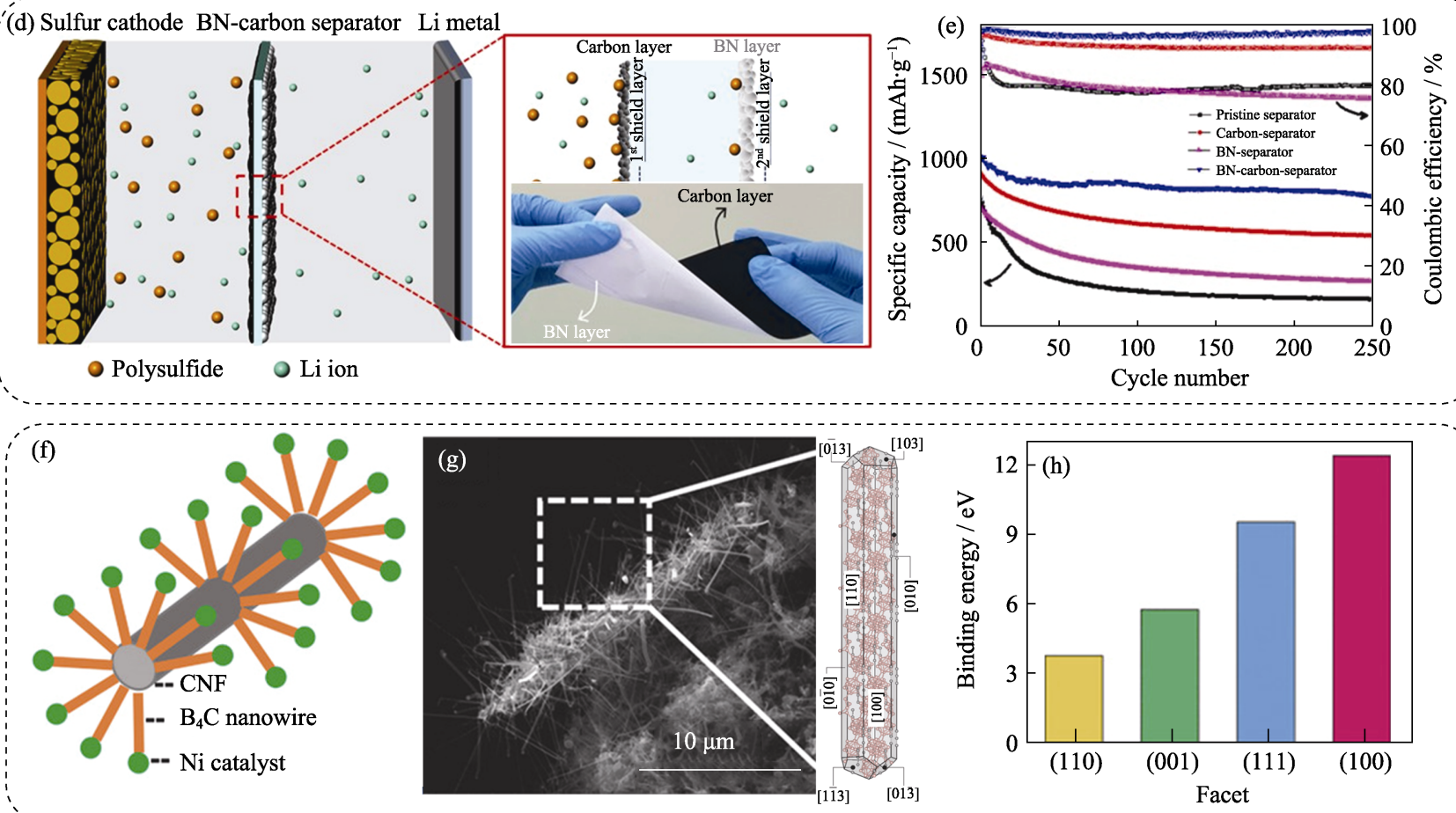

图 6 (a)v-BN 纳米片 TEM 照片及结构缺陷示意图 ${ }^{[78]} ;(\mathrm{b}) \mathrm{g}-\mathrm{C}_{3} \mathrm{~N}_{4} / \mathrm{BN} /$ 石墨烯复合离子笁结构示意图及(c)对应电池的循环 性能 ${ }^{[80]}$; (d) BN/Celgard/碳的三明治结构隔膜示意图和光学照片, 及其(e)电池循环性能 ${ }^{[83]}$; (f)B ${ }_{4} \mathrm{C} @ \mathrm{CNF}$ 的结构示意图,

$$
\mathrm{B}_{4} \mathrm{C} @ \mathrm{CNF} \text { 的 }(\mathrm{g}) \mathrm{SEM} \text { 照片和 } \mathrm{B}_{4} \mathrm{C} \text { 纳米线模型及其(h)不同晶面对 } \mathrm{Li}_{2} \mathrm{~S}_{4} \text { 的吸附能 }{ }^{[87]}
$$

Fig. 6 (a) TEM image and schematic atomic structure of $\mathrm{v}-\mathrm{BN}^{[78]}$; (b) Scheme of $\mathrm{g}_{-} \mathrm{C}_{3} \mathrm{~N}_{4} / \mathrm{BN} /$ graphene composite ion-sieve and (c) the corresponding Li-S cell cycling performance ${ }^{[80]}$; (d) Schematic and optical image of BN/Celgard/carbon trilayer separator, and (e) the corresponding cell cycling performance ${ }^{[83]}$; (f) Scheme and (g) SEM image of $\mathrm{B}_{4} \mathrm{C} @ \mathrm{CNF}$ and the model of $\mathrm{B}_{4} \mathrm{C}$ nanowire, (h) $\mathrm{Li}_{2} \mathrm{~S}_{4}$ adsorption energies on different facets of $\mathrm{B}_{4} \mathrm{C}^{[87]}$ 


\section{3 结论}

综上所述, 锂硫电池因其多电子转移反应具有 较高的理论能量密度, 然而其转换反应机制以及活 性材料本征的弱导电性阻碍了优势的发挥。硼基材 料具有独特的理化特征和电化学性质, 其针对性设 计与合理应用是缓解锂硫电池穿梭效应，提升反应 动力学和可逆性的有效途径, 近年来发展势头迅 猛。然而, 嗍基材料在锂硫电池中的研究和应用仍 处于起步阶段, 材料结构设计及其对电池电化学反 应过程的作用机制均有待进一步开发和探索。结合 材料特性以及上述研究进展, 作者认为, 锂硫电池 中嗍基材料的今后发展应更加关注以下几个方向:

1)材料合成。合成制备是上述嗍基材料面临的 共性难题，亟需发展更加简单、温和、高效的材料 制备方法, 为机理研究和应用推广提供材料基础。 其中，液相还原法制备无定型金属嗍化物是较有潜 力的发展方向，同时借鉴其优势和经验，探索和发 展基于溶剂热或熔融盐法的合成路径亦可能为硼基 材料的制备提供新的思路。此外，在硼化物的制备 过程中需格外注意对纳米结构及其稳定性的控制和 设计，以配合锂硫电池界面反应特性的需求。

2)机理探索。嗍基材料具有独特而丰富的表面 化学特征, 应着重结合原位表征手段, 深入研究硼 基材料和多硫化物间的主客体相互作用方式，特别 需要注意的是表面不可逆硫化、自身电化学氧化还 原等情况, 揭示其吸附和催化能力的决定性结构因 素, 为材料的针对性设计和开发提供理论指导和依 据。此外，对于颇具代表性的非晶态金属硼化物而 言, 需要格外关注无定型和晶相嗍化物在微观结构 及相关理化性质上的区别, 配合发展相应的结构解 析和性质表征分析技术，避免单纯基于晶态结构推 断非晶态材料与多硫化锂及其反应过程的作用关 系。

3)性能评估。优化材料和电池评估体系，在提高 硫面载量同时，应更加注重调控电极的厚度和孔隙 率等关键参数，同步提升电极的质量和体积能量密 度。此外, 进一步考察低电解液用量 $\left(E / S<5 \mathrm{~mL} \cdot \mathrm{g}^{-1} \mathrm{~s}\right)$ 、 低负/正极容量比 $(\mathrm{N} / \mathrm{P}<2)$ 条件下的电化学性质，同 时探索从实验室扣式电池到实际生产的柱状或软包 装电池等构架的放大效应及相关科学和工程问题, 对电池水平的性能竞争力做合理、综合的评估，为 锂硫电池的商业化发展提供指导和借鉴。

总之，本文围绕硼基材料，综述了硼烯、嗍原子 掺杂碳、金属硼化物及非金属硼化物在锂硫电池体
系中的最新研究进展，希望能给予同仁借鉴和启发， 拓展嗍基材料在新能源领域的开发和应用，以及推 动锂硫电池的实用化发展。

\section{参考文献:}

[1] DUNN B, KAMATH H, TARASCON J M. Electrical energy storage for the grid: a battery of choices. Science, 2011, 334(6058): 928-935.

[2] ARICO A S, BRUCE P, SCROSATI B, et al. Nanostructured materials for advanced energy conversion and storage devices. Nature Materials, 2005, 4(5): 366-377.

[3] LIANG Y R, ZHAO C Z, YUAN $\mathrm{H}$, et al. A review of rechargeable batteries for portable electronic devices. InfoMat, 2019, 1(1): 6-32.

[4] GOODENOUGH J B, PARK K S. The Li-ion rechargeable battery: a perspective. Journal of the American Chemical Society, 2013, 135(4): 1167-1176.

[5] TARASCON J M, ARMAND M. Issues and challenges facing rechargeable lithium batteries. Nature, 2011, 414: 171-179.

[6] JIN G Y, HE H C, WU J, et al. Cobalt-doped hollow carbon framework as sulfur host for the cathode of lithium sulfur battery. Journal of Inorganic Materials, 2021, 36(2): 203-209.

[7] FANG R, ZHAO S Y, SUN Z H, et al. More reliable lithium-sulfur batteries: tatus, solutions and prospects. Advanced Materials, 2017, 29(48): 1606823 .

[8] HU J J, LI G R, GAO X P. Current status, problems and challenges in lithium-sulfur batteries. Journal of Inorganic Materials, 2013, 28(11): 1181-1186.

[9] LI G R, WANG S, ZHANG Y N, et al. Revisiting the role of polysulfides in lithium-sulfur batteries. Advanced Materials, 2018, 30(22): 1705590.

[10] PENG H J, HUANG J Q, ZHANG Q. A review of flexible lithium-sulfur and analogous alkali metal-chalcogen rechargeable batteries. Chemical Society Reviews, 2017, 46(17): 5237-5288.

[11] JANA M, XU R, CHENG X B, et al. Rational design of two-dimensional nanomaterials for lithium-sulfur batteries. Energy \& Environmental Science, 2020, 13(4): 1049-1075.

[12] HE J R, MANTHIRAM A. A review on the status and challenges of electrocatalysts in lithium-sulfur batteries. Energy Storage Materials, 2019, 20: 55-70.

[13] SEH Z W, SUN Y M, ZHANG Q F, et al. Designing high-energy lithium-sulfur batteries. Chemical Society Reviews, 2016, 45(20): $5605-5634$.

[14] JI X L, EVERS S, BLACK R, et al. Stabilizing lithium-sulphur cathodes using polysulphide reservoirs. Nature Communications, 2011, 2: 325

[15] ZHANG Z, KONG L L, LIU S, et al. A high-efficiency sulfur/ carbon composite based on 3D graphene nanosheet@carbon nanotube matrix as cathode for lithium-sulfur battery. Advanced Energy Materials, 2017, 7(11): 1602543.

[16] XU W C, PAN X X, MENG X, et al. A conductive sulfur-hosting material involving ultrafine vanadium nitride nanoparticles for high-performance lithium-sulfur battery. Electrochimica Acta, 2020, 331: 135287

[17] LIU Y T, LIU S, LI G R, et al. High volumetric energy density sulfur cathode with heavy and catalytic metal oxide host for lithium-sulfur battery. Advanced Science, 2020, 7(12): 1903693.

[18] CHEN H H, XIAO Y W, CHEN C, et al. Conductive MOF modified separator for mitigating the shuttle effect of lithiumsulfur battery through a filtration method. ACS Applied Materials 
\& Interfaces, 2019, 11(12): 11459-11465.

[19] YOO J, CHO S J, JUNG G Y, et al. COF-net on CNT-net as a molecularly designed, hierarchical porous chemical trap for polysulfides in lithium-sulfur batteries. Nano Letters, 2016, 16(5): 3292-3300.

[20] HU Y, LIU C. Introduction of 1,2-migration for organoboron compounds. University Chemistry, 2019, 34(12): 39-44.

[21] SOREN K M, SUNING W. Boron-based stimuli responsive materials. Chemical Society Reviews, 2019, 48(13): 3537-3549.

[22] HUANG Z G, WANG S N, DEWHURST R D, et al. Boron: its role in energy-related processes and applications. Angewandte Chemie International Edition, 2020, 59(23): 8800-8816.

[23] ZHU Y H, GAO S M, HOSMANE N S. Boron-enriched advanced energy materials. Inorganica Chimica Acta, 2017, 471: 577-586.

[24] KHAN K, TAREEN A K, ASLAM M, et al. Synthesis, properties and novel electrocatalytic applications of the 2D-borophene xenes. Progress in Solid State Chemistry, 2020, 59: 100283.

[25] RAO D W, LIU X J, YANG H, et al. Interfacial competition between a borophene-based cathode and electrolyte for the multiple-sulfide immobilization of a lithium sulfur battery. Journal of Materials Chemistry A, 2019, 7(12): 7092-7098.

[26] JIANG H R, SHYY W, LIU M, et al. Borophene and defective borophene as potential anchoring materials for lithium-sulfur batteries: a first-principles study. Journal of Materials Chemistry A, 2018, 6(5): 2107-2114.

[27] ZHANG C Y, HE Q, CHU W, et al. Transition metals doped borophene-graphene heterostructure for robust polysulfide anchoring: a first principle study. Applied Surface Science, 2020, 534: 147575 .

[28] ZHANG L, LIANG P, SHU H B, et al. Borophene as efficient sulfur hosts for lithium-sulfur batteries: suppressing shuttle effect and improving conductivity. Journal of Physical Chemistry $C$, 2017, 121(29): 15549-15555.

[29] GRIXTI S, MUKHERJEE S, SINGH C V. Two-dimensional boron as an impressive lithium-sulphur battery cathode material. Energy Storage Materials, 2018, 13: 80-87.

[30] MANNIX A J, ZHOU X F, KIRALY B, et al. Synthesis of borophenes: anisotropic, two-dimensional boron polymorphs. Science, 2015, 350(6267): 1513-1516.

[31] FENG B J, ZHANG J, ZHONG Q, et al. Experimental realization of two-dimensional boron sheets. Nature Chemistry, 2016, 8(6): 564-569.

[32] PARAKNOWITSCH J P, THOMAS A. Doping carbons beyond nitrogen: an overview of advanced heteroatom doped carbons with boron, sulphur and phosphorus for energy applications. Energy \& Environmental Science, 2013, 6(10): 2839-2855.

[33] WANG H B, MAIYALAGAN T, WANG X. Review on recent progress in nitrogen-doped graphene: synthesis, characterization, and its potential applications. ACS Catalysis, 2012, 2(5): 781-794.

[34] XIE Y, MENG Z, CAI T W, et al. Effect of boron-doping on the graphene aerogel used as cathode for the lithium sulfur battery. ACS Applied Materials \& Interfaces, 2015, 7(45): 25202-25210.

[35] SHI P C, WANG Y, LIANG X, et al. Simultaneously exfoliated boron-doped graphene sheets to encapsulate sulfur for applications in lithium-sulfur batteries. ACS Sustainable Chemistry \& Engineering, 2018, 6(8): 9661-9670.

[36] YANG L J, JIANG S J, ZHAO Y, et al. Boron-doped carbon nanotubes as metal-free electrocatalysts for the oxygen reduction reaction. Angewandte Chemie International Edition, 2011, 50(31): 7132-7135.

[37] AI W, LI J W, DU Z Z, et al. Dual confinement of polysulfides in boron-doped porous carbon sphere/graphene hybrid for advanced
Li-S batteries. Nano Research, 2018, 11(9): 4562-4573.

[38] YANG C P, YIN Y X, YE H, et al. Insight into the effect of boron doping on sulfur/carbon cathode in lithium-sulfur batteries. ACS Applied Materials \& Interfaces, 2014, 6(11): 8789-8795.

[39] XU C X, ZHOU H H, FU C P, et al. Hydrothermal synthesis of boron-doped unzipped carbon nanotubes/sulfur composite for high-performance lithium-sulfur batteries. Electrochimica Acta, 2017, 232: 156-163.

[40] HAN P, MANTHIRAM A. Boron- and nitrogen-doped reduced graphene oxide coated separators for high-performance Li-S batteries. Journal of Power Sources, 2017, 369: 87-94.

[41] HOU T Z, CHEN X, PENG H J, et al. Design principles for heteroatom-doped nanocarbon to achieve strong anchoring of polysulfides for lithium-sulfur batteries. Small, 2016, 12(24): 3283-3291.

[42] XIONG D G, ZHANG Z, HUANG X Y, et al. Boosting the polysulfide confinement in $\mathrm{B} / \mathrm{N}$-codoped hierarchically porous carbon nanosheets via Lewis acid-base interaction for stable Li-S batteries. Journal of Energy Chemistry, 2020, 51: 90-100.

[43] YUAN S Y, BAO J L, WANG L N, et al. Graphene-supported nitrogen and boron rich carbon layer for improved performance of lithium-sulfur batteries due to enhanced chemisorption of lithium polysulfides. Advanced Energy Materials, 2016, 6(5): 1501733.

[44] CHEN L, FENG J R, ZHOU H H, et al. Hydrothermal preparation of nitrogen, boron co-doped curved graphene nanoribbons with high dopant amounts for high-performance lithium sulfur battery cathodes. Journal of Materials Chemistry A, 2017, 5(16): 7403-7415.

[45] JIN C B, ZHANG W K, ZHUANG Z Z, et al. Enhanced sulfide chemisorption using boron and oxygen dually doped multi-walled carbon nanotubes for advanced lithium-sulfur batteries. Journal of Materials Chemistry A, 2017, 5(2): 632-640.

[46] ULLAH S, DENIS P A, SATO F. Unusual enhancement of the adsorption energies of sodium and potassium in sulfur-nitrogen and silicon-boron codoped graphene. ACS Omega, 2018, 3(11): 15821-15828.

[47] ZHANG Z, XIONG D G, SHAO A H, et al. Integrating metallic cobalt and N/B heteroatoms into porous carbon nanosheets as efficient sulfur immobilizer for lithium-sulfur batteries. Carbon, 2020, 167: 918-929.

[48] WANG P, KUMAR R, SANKARAN E M, et al. Vanadium diboride $\left(\mathrm{VB}_{2}\right)$ synthesized at high pressure: elastic, mechanical, electronic, and magnetic properties and thermal stability. Inorganic Chemistry, 2018, 57(3): 1096-1105.

[49] HE G J, LING M, HAN X Y, et al. Self-standing electrodes with core-shell structures for high-performance supercapacitors. Energy Storage Materials, 2017, 9: 119-125.

[50] WANG C C, AKBAR S A, CHEN W, et al. Electrical properties of high-temperature oxides, borides, carbides, and nitrides. Journal of Materials Science, 1995, 30(7): 1627-1641.

[51] XIAO Z B, YANG Z, ZHANG L J, et al. Sandwich-type $\mathrm{NbS}_{2} @ \mathrm{~S} @ I$-doped graphene for high-sulfur-loaded, ultrahigh-rate, and long-life lithium sulfur batteries. ACS Nano, 2017, 11(8): 8488-8498.

[52] WANG L J, LIU F H, ZHAO B Y, et al. Carbon nanobowls filled with $\mathrm{MoS}_{2}$ nanosheets as electrode materials for supercapacitors. ACS Applied Nano Materials, 2020, 3(7): 6448-6459.

[53] BALACH J, LINNEMANN J, JAUMANN T, et al. Metal-based nanostructured materials for advanced lithium-sulfur batteries. Journal of Materials Chemistry A, 2018, 6(46): 23127-23168.

[54] BEN-DOR L, SHIMONY Y. Crystal structure, magnetic susceptibility and electrical conductivity of pure and $\mathrm{NiO}$-doped $\mathrm{MoO}_{2}$ and $\mathrm{WO}_{2}$. Materials Research Bulletin, 1974, 9(6): 837-44. 
[55] SAMSONOV G. 难熔化合物手册. 北京: 中国工业出版社, 1965: $1-147$.

[56] FENG L S, QUN C X, LIN M Y, et al. Nb-based oxides as anode materials for lithium ion batteries. Progress in Chemistry, 2015, 27(2/3): 297-309.

[57] TAO Q, MA S L, CUI T, et al. Structures and properties of functional transition metal borides. Acta Physica Sinica, 2017, 66(3): 036103.

[58] SHEN Y F, XU C, HUANG M, et al. Research advances of boron clusters,borane and metal-doped boron compounds. Progress in Chemistry, 2016, 28(11): 1601-1614.

[59] GUPTA S, PATEL M K, MIOTELLO A, et al. Metal boride-based catalysts for electrochemical water-splitting: a review. Advanced Functional Materials, 2020, 30(1): 1906481.

[60] WU F, WU C. New secondary batteries and their key materials based on the concept of multi-electron reaction. Chinese Science Bulletin, 2014, 59(27): 3369-3376.

[61] GUAN B, FAN L S, WU X, et al. The facile synthesis and enhanced lithium-sulfur battery performance of an amorphous cobalt boride $\left(\mathrm{Co}_{2} \mathrm{~B}\right) @$ graphene composite cathode. Journal of Materials Chemistry A, 2018, 6(47): 24045-24049.

[62] GUAN B, ZHANG Y, FAN L S, et al. Blocking polysulfide with $\mathrm{Co}_{2} \mathrm{~B} @$ CNT via "synergetic adsorptive effect" toward ultrahighrate capability and robust lithium-sulfur battery. ACS Nano, 2019, 13(6): $6742-6750$

[63] GUAN B, SUN X, ZHANG Y, et al. The discovery of interfacial electronic interaction within cobalt boride@MXene for high performance lithium-sulfur batteries. Chinese Chemical Letters, 2020, 32(7): 2249-2253.

[64] BASU B, RAJU GSURI A. Processing and properties of monolithic $\mathrm{TiB}_{2}$ based materials. International Materials Reviews, 2006, 51(6): 352-374.

[65] LI C C, LIU X B, ZHU L, et al. Conductive and polar titanium boride as a sulfur host for advanced lithium-sulfur batteries. Chemistry of Materials, 2018, 30(20): 6969-6977.

[66] LI Z J, JIANG H R, LAI N C, et al. Designing effective solventcatalyst interface for catalytic sulfur conversion in lithium-sulfur batteries. Chemistry of Materials, 2019, 31(24): 10186-10196.

[67] JIN L M, NI J, SHEN C, et al. Metallically conductive $\mathrm{TiB}_{2}$ as a multi-functional separator modifier for improved lithium sulfur batteries. Journal of Power Sources, 2020, 448: 227336.

[68] WU R, XU H K, ZHAO Y W, et al. Borophene-like boron subunits-inserted molybdenum framework of $\mathrm{MoB}_{2}$ enables stable and quick-acting $\mathrm{Li}_{2} \mathrm{~S}_{6}$-based lithium-sulfur batteries. Energy Storage Materials, 2020, 32: 216-224.

[69] HE J R, BHARGAV A, MANTHIRAM A. Molybdenum boride as an efficient catalyst for polysulfide redox to enable high-energydensity lithium-sulfur batteries. Advanced Materials, 2020, 32(40): 2004741.

[70] PANG Q, KWOK C Y, KUNDU D, et al. Lightweight metallic $\mathrm{MgB}_{2}$ mediates polysulfide redox and promises high-energy-density lithium-sulfur batteries. Joule, 2019, 3(1): 136-148.

[71] YU T T, GAO P F, ZHANG Y, et al. Boron-phosphide monolayer as a potential anchoring material for lithium-sulfur batteries: a first-principles study. Applied Surface Science, 2019, 486: 281-286.

[72] JANA S, THOMAS S, LEE C H, et al. $\mathrm{B}_{3} \mathrm{~S}$ monolayer: prediction of a high-performance anode material for lithium-ion batteries. Journal of Materials Chemistry A, 2019, 7(20): 12706-12712.

[73] SUN C, HAI C X, ZHOU Y, et al. Highly catalytic boron nitride nanofiber in situ grown on pretreated ketjenblack as a cathode for enhanced performance of lithium-sulfur batteries. ACS Applied Energy Materials, 2020, 3(11): 10841-10853.

[74] ARENAL R, LOPEZ BEZANILLA A. Boron nitride materials: an overview from $0 \mathrm{D}$ to $3 \mathrm{D}$ (nano)structures. Wiley Interdisciplinary Reviews-Computational Molecular Science, 2015, 5(4): 299-309.

[75] JIANG X F, WENG Q H, WANG X B, et al. Recent progress on fabrications and applications of boron nitride nanomaterials: a review. Journal of Materials Science and Technology, 2015, 31(6): 589-598.

[76] PRAKASH A, NEHATE S D, SUNDARAM K B. Boron carbon nitride based metal-insulator-metal UV detectors for harsh environment applications. Optics Letters, 2016, 41(18): 4249-4252.

[77] ZHAO Y M, YANG L, ZHAO J X, et al. How to make inert boron nitride nanosheets active for the immobilization of polysulfides for lithium-sulfur batteries: a computational study. Physical Chemistry Chemical Physics, 2017, 19(28): 18208-18216.

[78] YI Y K, LI H P, CHANG H H, et al. Few-layer boron nitride with engineered nitrogen vacancies for promoting conversion of polysulfide as a cathode matrix for lithium-sulfur batteries. Chemistry, 2019, 25(34): 8112-8117.

[79] HE B, LI W C, ZHANG Y, et al. Paragenesis BN/CNTs hybrid as a monoclinic sulfur host for high rate and ultra-long life lithiumsulfur battery. Journal of Materials Chemistry A, 2018, 6(47): 24194-24200.

[80] DENG D R, BAI C D, XUE F, et al. Multifunctional ion-sieve sonstructed by 2D materials as an interlayer for Li-S batteries. ACS Applied Materials \& Interfaces, 2019, 11(12): 11474-11480.

[81] SUN K, GUO P Q, SHANG X N, et al. Mesoporous boron carbon nitride/graphene modified separators as efficient polysulfides barrier for highly stable lithium-sulfur batteries. Journal of Electroanalytical Chemistry, 2019, 842: 34-40.

[82] FAN Y, YANG Z, HUA W X, et al. Functionalized boron nitride nanosheets/graphene interlayer for fast and long-life lithium-sulfur batteries. Advanced Energy Materials, 2017, 7(13): 1602380.

[83] KIM P J H, SEO J, FU K, et al. Synergistic protective effect of a $\mathrm{BN}$-carbon separator for highly stable lithium sulfur batteries. NPG Asia Materials, 2017, 9(4): e375.

[84] PRAMANICK A, DEY P P, DAS P K. Microstructure, phase and electrical conductivity analyses of spark plasma sintered boron carbide machined with WEDM. Ceramics International, 2020, 46(3): $2887-2894$.

[85] YEGANEH M, SARAF H H, KAFI F, et al. First principles investigation of vibrational, electronic and optical properties of graphene-like boron carbide. Solid State Communications, 2020, 305: 113750

[86] CHANG Y K, SUN X H, MA M D, et al. Application of hard ceramic materials $\mathrm{B}_{4} \mathrm{C}$ in energy storage: design $\mathrm{B}_{4} \mathrm{C} @ \mathrm{C}$ core-shell nanoparticles as electrodes for flexible all-solid-state microsupercapacitors with ultrahigh cyclability. Nano Energy, 2020, 75: 104947.

[87] LUO L, CHUNG S H, ASL H Y, et al. Long-life lithium-sulfur batteries with a bifunctional cathode substrate configured with boron carbide nanowires. Advanced Materials, 2018, 30(39): 1804149.

[88] SONG N N, GAO Z, ZHANG Y Y, et al. $\mathrm{B}_{4} \mathrm{C}$ nanoskeleton enabled, flexible lithium-sulfur batteries. Nano Energy, 2019, 58: 30-39.

[89] ZHANG R H, CHI C, WU M C, et al. A long-life Li-S battery enabled by a cathode made of well-distributed $\mathrm{B}_{4} \mathrm{C}$ nanoparticles decorated activated cotton fibers. Journal of Power Sources, 2020, 451: 227751 